Sociologie et sociétés

\title{
Le rétablissement du carnaval à Berlin
}

Performance publique et politique dans la nouvelle capitale

allemande

\author{
Re-implanting the Carnival in Berlin. \\ Public Performance and Politics in the New German Capital
}

\section{Franziska Becker}

Volume 37, numéro 1, printemps 2005

Le spectacle des villes

The Spectacle of Cities

URI : https://id.erudit.org/iderudit/012275ar

DOI : https://doi.org/10.7202/012275ar

Aller au sommaire du numéro

\section{Éditeur(s)}

Les Presses de l'Université de Montréal

ISSN

0038-030X (imprimé)

1492-1375 (numérique)

Découvrir la revue

Citer cet article

Becker, F. (2005). Le rétablissement du carnaval à Berlin : performance publique et politique dans la nouvelle capitale allemande. Sociologie et sociétés, 37(1), 35-54. https://doi.org/10.7202/012275ar
Résumé de l'article

Se penchant sur les multiples tentatives de réintroduction du carnaval de rue à Berlin, cet article s'intéresse à la volonté de reconstruire Berlin comme lieu et théâtre de l'Allemagne réunifiée en opérant certaines transformations symboliques issues de divers débats sur la façon dont la ville peut devenir cette capitale. À travers une perspective comparative historique, l'article analyse les campagnes de marketing politico-culturelles de réinvention ainsi que les diverses mises en scène innovatrices de performances culturelles pour donner au carnaval d'origine rhénane un caractère berlinois, dans deux contextes historiques et sociopolitiques différents, soit celui des années 1950 et celui des années 2000. L'article démontre que la capacité de ces mises en scène à s'établir dans le cadre des processus de transformation de la ville postfordiste est soumise à un processus complexe de négociations entre les groupes d'acteurs participant à la préparation et à l'évaluation de telles performances symboliques. 


\section{FRA NZISKA BECKER}

Institut für Europäische Ethnologie/Kulturwissenschaft

Philipps-Universität Marburg

Biegenstrasse 9

35037 Marburg

Courriel : beckerf@staff.uni-marburg.de

TRADUCTION : DAVID OUELLET

$\mathrm{B}$

ERLIN, 1952. «Le carnaval aussi doit s'apprendre », titre le Tagesspiegel du 27 février:

Quiconque craignait voir les Berlinois se transformer en Rhénans a dû se détromper (...) tandis que passait dans les rues le premier défilé berlinois du Lundi des Roses. Certes, il y avait une cohue dangereuse dans le métro: des grappes de gens, comme on en avait vues à satiété pendant la guerre et l'après-guerre, s'accrochaient aux voitures de tram; les ruines le long des grandes artères étaient occupées jusqu'au troisième étage (...) par les badauds; et des masses de gens se tenaient debout sur les amas de débris qui durent faire office de tribunes (...) Jusquelà, les apparences extérieures étaient tout à fait carnavalesques. Mais le Berlinois n'est pas dupe, et dans notre climat, on ne cueille pas les lauriers prématurément; c'est pourquoi l'atmosphère générale était d'un scepticisme enjoué. Trois personnes légèrement animées qui essayaient près de moi de galvaniser l'atmosphère par la force furent certes considérées avec bienveillance, bien que très sensiblement comme des étrangers. Les autochtones n'exerçaient aucune pression sur leur tempérament naturel: ils étaient à l'expectative (...). Les gens se comportaient plutôt comme ces visiteurs de zoo, qu'Anton Glassbrenner a décrits comme: modérément curieux, un peu déconcertés, un peu amusés, mais en tout cas étaient tous du même avis: «Une chance qu'il y a une grille entre nous.» Le grillage n'est pas tombé en ce Lundi des Roses.» 
Si ce n'était des ruines et des amas de débris, ces symboles de l'après-guerre qui forment le décor du premier défilé du carnaval de Berlin, les impressions journalistiques auraient bien pu dater de 2001, alors que pour la première fois depuis les années 1950, un défilé de carnaval classique passait de nouveau dans les rues; cette fois-ci, il ne passa pas dans les anciens districts de l'ouest, mais dans le nouveau quartier gouvernemental au centre de la ville. La teneur de la presse ressemble à celle de 1952 :

Avenue Unter den Linden, les Berlinois accueillent le défilé avec un scepticisme curieux. La plupart sont debout au bord de la rue, restent dans l'expectative et lèvent le regard vers les chars allégoriques modestement décorés. Seule une minorité des 100 ooo spectateurs, selon les informations de la police, est venue déguisée.

Comme au début des années 1950, le défilé du carnaval semble n’avoir connu qu'une résonance modérée en 2001. Près de 50 ans plus tard, on assiste à la tentative renouvelée d'introduire le carnaval de rue à Berlin. Que les deux tentatives aient eu lieu à des périodes de bouleversement, où Berlin était en phase de constitution, présente un autre parallèle: une première tentative à la suite du nazisme, de la guerre, de la division de l'Allemagne, du blocus de Berlin, de la réforme monétaire et de l'ascension de Bonn au rang de capitale; une seconde tentative après la fin de la guerre froide, la réunification des deux États allemands et le déménagement du gouvernement de Bonn à Berlin, à une époque où la ville se cherche un nouveau profil de capitale et de métropole internationale. Alors qu'à l'époque de l'après-guerre, le défilé du carnaval fut suspendu après seulement quelques années, il reste à voir si aujourd'hui le défilé deviendra un de ces grands événements berlinois comme la Love Parade, Christopher-Street-Day ou le défilé multiculturel, le Carnaval des cultures. Déjà en février 2002, plus de 200000 spectateurs auraient jalonné le passage du deuxième défilé du carnaval désormais devenu «classique». Des journalistes bienveillants ont même voulu y voir le signe que la nouvelle capitale pourrait devenir le bastion du carnaval.

En juin 1991, le parlement allemand décida par une mince majorité de déplacer le siège du gouvernement de Bonn à Berlin. Depuis, on construit surtout dans le «nouveau Mitte $^{1} »$ de Berlin. De nouveaux édifices gouvernementaux tels que la chancellerie et les bureaux des députés parlementaires y ont pris naissance, des ambassades y ont ouvert leurs résidences, la Potsdamer Platz y a été réaménagée et d'autres projets, sur Alexanderplatz par exemple, sont imminents tandis que des lieux commémoratifs comme le mémorial de l'Holocauste y sont actuellement érigés. Toutefois, un processus de restructuration se déroule en parallèle qui ne se conclura en aucun cas par la finition des seuls immeubles ou le réaménagement de places centrales. Il s'agit bien plus de restructurer Berlin comme lieu et théâtre de la « République de Berlin ». On entend par là le processus de transformation symbolique d'un espace urbain, de ses édifices, de sa culture et de sa société en scène

1. Mitte : quartier du centre historique de Berlin (note du traducteur). 
de politique nationale et internationale ${ }^{2}$. Car le déménagement du gouvernement et l'ascension de Berlin au rang de capitale, avec tout ce que cela comprend de phénomènes concomitants économiques, politiques et culturels, affectent non seulement les champs d'action politiques les plus variés de la ville, mais aussi, en essence, sa structure sociale et culturelle. Or, ceci provoque forcément des conflits; la recodification de l'espace urbain et la reconstitution de la société urbaine sont accompagnées de débats sociaux étendus sur la manière de façonner Berlin en tant que «capitale représentative» de l'Allemagne réunifiée et métropole de services ouverte sur le monde. Dans ce contexte, l'espace urbain est utilisé tout aussi bien par des acteurs politiques que par différents groupes sociaux pour faire valoir leurs intérêts. Dans la foulée de ce processus de transformation symbolique, on assiste à une réinvention et à une mise en scène innovatrices des formes de cultural performance ${ }^{3}$, rendues ainsi utiles pour la représentation du «nouveau Berlin» dans l'espace public. La tangibilité et l'expérimentabilité de ces représentations en font d'ailleurs un important terrain de lutte où se jouent les conflits politiques et sociaux. Ainsi, les cultural performances peuvent autant servir de stratégie politique pour inscrire dans l'espace urbain des interprétations et des significations que pour procurer la possibilité d'articuler des positions politiques adverses. Les performances sont, en ce sens, des représentations culturelles qui développent dans l'espace social leur propre puissance d'action ${ }^{4}$.

Le carnaval de rue est une telle public performance ${ }^{5}$ arrivée à Berlin dans le sillon du déménagement du gouvernement de Bonn avec ses parlementaires, ses fonctionnaires ministériels et ses employés. Que la classe politique de l' " ancienne République de Bonn » se soit non seulement transportée dans la nouvelle capitale, mais ait aussi emporté avec elle ses mœurs du Rhin à la Spree, déclencha un violent débat qui s'est surtout déroulé dans

2. Intitulé «Scènes du pouvoir. De la mise en scène du "quartier gouvernemental" comme espace de représentation », ce projet de recherche subventionné par la Deutsche Forschungsgemeinschaft (DFG) fut entrepris en 1998 à l'Institut für Europäische Ethnologie de l'Université Humboldt à Berlin et analysait le processus de transformation symbolique de Berlin dans une perspective ethnologique. Voir Franziska Becker et Beate Binder, " "Bühnen der Macht". Stadtethnologische Perspektiven auf die Hauptstadtwerdung Berlins », dans Humboldt Spektrum, vol. 8, nº 3-4/2001, p. 112-119; Franziska Becker et Beate Binder, « Hauptstadt-Rituale. Cultural performances und ihre Autorisierung im öffentlichen Raum», dans Erika Fischer Lichte et al. (dir.), «Ritualität und Grenze», Theatralität, vol. 5, Tübingen, 2003, p. 224-244.

3. En anglais dans le texte (note du traducteur). La notion est attribuée à Milton Singer; Don Handelman l'a reprise conceptuellement dans l'analyse d'événements publics au sein de sociétés dites complexes. Voir Milton Singer, «Search for Great Traditions in Cultural performance», dans l'ouvrage du même auteur When Great Tradition Modernizes: An Anthropological Approach to Indian Civilization, Londres 1972, p. 67-80 ; Don Handelman, Models and Mirrors. Toward an Anthropology of Public Events, Cambridge, 1990.

4. Cette perspective est le produit des récentes théories de la performance et des rituels qui ont relativisé la conception d'événements publics ritualisés comme miroirs, voire symboles clés d'une société entière gisant à l'extérieur d'elle-même. L'analyse des combats de coqs au Bali par Clifford Geertz, où le combat de coqs est décrit comme symbole clé pour toute la société, est considérée paradigmatique pour cette conception. (Voir Clifford Geertz, "Deep Play: Bemerkungen zum Balinesischen Hahnenkampf», dans l'ouvrage du même auteur Dichte Beschreibung, Francfort-sur-le-Main 1993, p. 202-26o.) Au lieu de cela, je soulignerai ici le caractère formatif et constructif des cultural performances pour les réalités sociales. Voir F. G. Bailey, «Cultural Performance, Authenticity, and Second Nature», dans David Parkin et al. (dir.), The Politics of Culture Performance, Oxford, 1996, p. 1-17.

5. En anglais dans le texte (note du traducteur). 
les médias. Il s'agissait essentiellement de savoir si le carnaval était légitime en tant que rituel d'établissement des «Bonnois à Berlin » et si ce spectacle avait la moindre chance de s'enraciner à Berlin.

Dans ce qui suit, j'aborderai l'amorcement du déménagement du carnaval à Berlin dans deux contextes historiques et sociopolitiques différents. Je souhaite ainsi révéler comment cette public performance est utilisée pour la présentation d'élites politiques et économiques et à quelles stratégies de mise en scène ces élites ont recours dans le processus de l'Invention of Tradition ${ }^{6}$ pour prêter au carnaval d'origine rhénane un caractère berlinois. Or, qu'est-ce qui détermine l'implantation de pareilles mises en scène et leur enracinement durable dans l'espace social? J'aborderai cette question dans un second temps au moyen d'une rétrospective des années 1950.

À cette époque aussi ce sont les élites économiques et politiques qui essayèrent d'implanter le carnaval comme spectacle public dans une phase de changements urbains profonds. Quelles stratégies de représentation poursuivaient-elles à cet effet et pourquoi ces stratégies échouèrent-elles? Seule une comparaison diachronique, selon ma thèse, fournit des réponses au-delà du cas concret à la question suivante: quels contextes culturels, politiques et sociaux permettent ou limitent l'établissement d'une cultural performance dans des phases de changements urbains profonds? Question qui, justement en considération de la "political economy of imageability ${ }^{7}$ » dans le cadre des processus de transformation de la ville postfordiste, pourrait être pertinente.

Des sociologues de la ville ont décrit par la formule «festivalisation de la politique urbaine » la manière dont, depuis les années 1980, les grands événements sont de plus en plus utilisés comme des instruments de politique urbaine ${ }^{8}$. Comme dans une campagne électorale, argent, individus et médias sont mobilisés en fonction d'un objectif défini le plus clairement possible pour faire la promotion de la ville comme emplacement porteur d'avenir, pour engendrer la croissance, attirer les investisseurs et être concurrentielle dans le cadre de la compétition européenne et internationale entre villes. Les grands événements ne rapportent pas seulement des revenus économiques, ils aiguillonnent la mise en scène médiatique de la ville et contribuent ainsi à sa revalorisation symbolique 9 . Toutefois, ceci se produit en règle générale, selon l'opinion prépondérante des sociologues urbains, "de haut en bas», c'est-à-dire sans donner aux acteurs locaux la possibilité de participer, si bien que les festivals apportent à toute ville les bénéfices du tourisme et

6. Eric Hobsbawn, «Introduction: Inventing Traditions», in Eric Hobsbawn et Terence Ranger (dir.), The Invention of Tradition, Cambridge, 1993, p. 1-15.

7. M. C. Boyer, «The Great Frame Up. Fantastic Appearance in Contemporary Spatial Politics », dans H. Liggett et D. C. Perry (dir.), Spatial Practice, Thousand Oaks, Sage Publications, 1995, p. 81-109.

8. Hartmut Häußermann et Walter Siebel, « Die Politik der Festivalisierung und die Festivalisierung der Politik. Große Ereignisse in der Stadtpolitik», dans leur ouvrage Festivalisierung der Stadtpolitik, Leviathan, numéro spécial 13/1993, p. 7-32.

9. La sociologue américaine Sharon Zukin a dégagé dans son ouvrage The Cultures of Cities le fonctionnement de l'économie symbolique et culturelle comme moyen d'encadrer l'espace framing space). Un des objectifs fondamentaux d'un tel chargement symbolique des espaces urbains au moyen d'emblèmes et d'événements culturels est le selling place, l'attraction de prestataires de services, de touristes et d'élites comme piliers de l'économie urbaine. Voir Sharon Zukin, The Cultures of Cities, Cambridge (MA), Blackwell, 1995, p. 7. 
l'occasion de polir son image, mais au prix de la disparition de l'identité locale. À l'encontre de cela nous soutiendrons, d'une part, la thèse que l'ancrage social « réussi », c'està-dire durable, d'une telle mise en scène dans l'espace urbain nécessite l'inclusion d'acteurs locaux. D'autre part, j'aborderai la thèse selon laquelle les campagnes de marketing politico-culturelles doivent s'orienter vers $\mathrm{l}^{\prime}$ " imaginaire de la ville ${ }^{10}$ » spécifique à chaque cas. C'est en ces termes que Rolf Lindner caractérise dans ses réflexions sur le point de départ d'une anthropologie de la ville (Anthropology of the City) le fonds d'images, de significations et d'imaginations d'une texture culturelle locale enracinée dans l'histoire qui marquerait l'ethos et l'habitus d'une ville ${ }^{11}$. L'ancrage d'une cultural performance dans le processus de transformation urbaine se mesure donc non seulement selon des critères économiques ou organisationnels, mais surtout selon la crédibilité de la mise en scène qui doit se rapporter au contexte local pour en tirer du sens. La crédibilité ou l'«authenticité» ne peuvent cependant pas être fabriquées exclusivement «de haut en bas» dans une logique stratégique et instrumentale; elles doivent plutôt être négociées entre divers regroupements d'acteurs impliqués dans la préparation, la réalisation et l'évaluation de grands événements : entre initiateurs, organisateurs, participants, spectateurs et médias ${ }^{12}$.

Passons donc au noyau anthropologique de la question en examinant dans un premier temps la mise en scène renouvelée du carnaval dans le contexte contemporain de la transformation de Berlin en ville capitale, puis, dans un second temps, par le biais d'une rétrospective des années 1950.

\section{ENTRE MULTICULTURALISME ET TRADITION: STRATÉGIES DE MISE EN SCÈNE DANS LE « NOUVEAU BERLIN »}

L’idée a «germé» dans un local du nouveau quartier gouvernemental non loin du Reichstag appelé «Mission permanente ${ }^{13}$ », un bistro de style rhénan dont la réputation dépasse

10. Rolf Lindner, "The Imaginary of the City», dans The Contemporary Study of Culture, édité par le ministère fédéral de la Science et des Transports et par le Centre international de recherche sur les sciences de la culture, Vienne, 1999a, p. 289-294.

11. Rolf Lindner, «Stadtkultur», dans Hartmut Häußermann (dir.), Großstadt. Soziologische Stichworte, Opladen, 1998, p. 256-262; du même auteur : «Die kulturelle Textur der Stadt », dans Das Magazin. Zeitschrift des Wissenschaftszentrums Nordrhein-Westfalen, vol. 10, 1999b, p. 34-35.

12. À propos du caractère complexe de la négociation d'événements ritualisés, voir Gerd Baumann, "Ritual implicates “Others" : Rereading Durkheim in a Plural Society», dans Daniel de Coppet (dir.), Understanding Rituals, Londres, 1992 ; E. L. Schieffelin, «Problematizing Performance», dans F. Hughes-Freeland (dir.) Ritual, Performance, Media, Londres, 1998, p. 194-207; Abner Cohen, Masquerade Politics. Explorations in the Structure of Urban Cultural Movements, Providence, 1993. R. L. Grimes a fait remarquer que la réflexion et l'évaluation d'un événement contribuent à construire cet événement; ceci vaut tant pour la perception que pour la reprise de l'événement. (R. L. Grimes, Ritual Criticism: Case Studies in its Practice. Essays on the Theory, Columbia, 1990.) Les médias en particulier ont un rôle à jouer dans les sociétés plurielles.

13. Emprunt du nom de l'ancienne représentation diplomatique ouest-allemande en RDA. Le local est situé rue Schiffbauer Damm à un coin de rue des institutions politiques et médiatiques : Reichstag, bureaux des députés, centre fédéral des conférences de presse, émetteurs de télévision et quelques centrales de partis politiques. 
Berlin, ouvert en 1997 par un éminent restaurateur bonnois et son compagnon spécialisé dans la réception de politiciens. Les deux restaurateurs avaient déménagé à Berlin à la remorque du gouvernement. En activant leurs réseaux de contacts dans le monde politique et médiatique, ils lancèrent une campagne d'image pour leur bistro qui reposait sur la folklorisation de la politique et du déménagement du gouvernement. Sous l'étiquette de gastronomie-spectacle, la politique bonnoise et les «Bonnois à Berlin» deviennent une valeur de divertissement à saveur culinaire ${ }^{14}$. Depuis, le bistro sert non seulement de «bassin de réception pour nostalgiques de Bonn ${ }^{15}$ ", mais aussi de lieu de rendez-vous pour les employés du Bundestag, les lobbyistes, les journalistes et les politiciens, et surtout de site touristique très fréquenté. C'est ici, dans ce lieu où politique semi-publique et intérêts économiques se mêlent, que la préparation du nouveau défilé de rue de la capitale s’amorce. À ses débuts, la « Mission permanente » était justement un terrain de recherche prisé de la presse locale et suprarégionale. Non seulement peut-on y découvrir «les Bonnois » et les interroger à propos de leurs sensibilités à l'endroit de Berlin, mais on peut aussi y faire la démonstration que l'arrivée de la nouvelle classe politique conduit à un enrichissement et à une revalorisation culturels de la nouvelle capitale. Même le Wallstreet Journal Europe titra un article sur le restaurant : "A Capital Question : Can Bonners Make Berlin a "Nice" Place?», où l'on lisait que les «Bonners don't just bring bureaucrats. They also bring beer [...], are importing their own food, too» (17 novembre 1999). La mise en scène folklorique des «Bonnois à Berlin » y a même promu la célébration annuelle d'un carnaval original de bistro rhénan et ainsi transformé le bistro en lieu authentique de la célébration des mœurs rhénanes. Ceci apporta au bistro et à ses exploiteurs de la publicité supplémentaire que les gastronomes - outre leurs réseaux de journalistes et de politiciens transplantés de Bonn à Berlin - pouvaient utiliser pour leur campagne de promotion du défilé du carnaval.

À l'échelle rhénane, du moins, on nota lors du premier défilé entièrement berlinois en février 2004 le rapport encore un peu gauche avec le genre de la part des spectateurs comme des acteurs: les gardes des quelque vingt associations de carnaval de l'est et de l'ouest de la ville qui défilaient gravement semblaient encore tout ignorer de la prestation publique et leur âge moyen était très élevé; de grands vides dans le déroulement du défilé, aucune musique d'ambiance continue et entraînante, trop peu de confettis, des spectateurs passifs qui rentraient la tête dans les épaules plutôt que d'attraper les bonbons au vol ; plusieurs véhicules décorés sobrement à des fins publicitaires étaient aussi de la partie ; l'habituel persiflage rhénan de la politique actuelle et les chars allégoriques transportant des silhouettes de politiciens en carton faisaient défaut. Les politiciens éminents, sur le plan régional comme fédéral, évitèrent le spectacle. Malgré sa route passant par le nouveau centre politique de la ville - depuis la Wilhelmstrasse, longeant Unter den Linden

14. Ceci touche surtout le décor du bistro : la couverture médiatique du débat parlementaire sur le déménagement du gouvernement est documentée dans les toilettes; on y trouve accrochées aux murs du bistro des photos d'éminents politiciens de l'époque de Bonn adoptant des poses amusantes, des symboles de la « réunification", voire de la «colonisation ouest-allemande», tel ce drapeau exhibant l'inscription «Apprendre de l'Allemand de l'Ouest signifie apprendre à vaincre ». À cela s'ajoute une cuisine typiquement rhénane célébrée dans des menus commentés.

15. Tageszeitung, 12 septembre 2000. 
jusqu'à l'hôtel de ville rouge ${ }^{16}$-, le défilé de rue était à tous égards apolitique. Pourtant, les quotidiens à grand tirage décrétèrent généralement qu'il s'agissait d'un succès, surtout en raison de l'affluence inattendue (environ 100 ooo personnes). Prise dans le contexte d'autres spectacles de métropole berlinois, l'affirmation que la «Love Parade des citoyens ${ }^{17}$ " était peut-être arrivée dans la nouvelle capitale semblait quelque peu ironique.

Le deuxième défilé, qui en 2002 prit son départ au Reichstag, présenta une image semblable, bien qu'on compta sensiblement plus de spectateurs (environ 200 000). Exception faite de quelques petites pancartes faisant allusion à l'euro, la satire politique fit cette fois aussi défaut. La résonance médiatique fit écho à la teneur de l'événement, bien que beaucoup moins que la première fois, car la presse locale et nationale avait déjà au préalable abondamment couvert la nouveauté d'un carnaval dans la capitale. Cette nouveauté faisait l'effet d' "œuvre missionnaire» de «Rhénans exilés » et fraîchement arrivés qui cherchaient à ressusciter une tradition interrompue en $1958^{18}$. Simultanément, des modèles argumentatifs sceptiques proches de ceux des années 1950 firent leur apparition, voulant que le carnaval soit étranger aux Berlinois et la possibilité de l'établir ici, douteuse.

Bien que le débat fût parfois d'une ironie tranchante, il n'en demeure pas moins qu'il comptait aussi une dimension résolument politique. Le carnaval était un symbole disputé quant à savoir si la nouvelle classe politique pouvait utiliser l'espace urbain pour la mise en scène de sa propre présence. Déterminer si les Bonnois modifiaient Berlin ou si l'inverse était vrai avait constitué une question centrale déjà avant le déménagement du gouvernement. Ainsi avait-on spéculé sur la question de savoir si la politique fédérale s'isolerait de la dure réalité de la vie berlinoise ou si «l'âpre ville de Berlin exercerait une pression plus forte à agir que l'idyllique ville de Bonn où les formes d'expression contestataire étaient plus bourgeoises et inoffensives qu'ici ${ }^{19}{ }^{\prime}$. Lorsque les Bonnois arrivèrent, la critique se fit surtout entendre à l'endroit de leur intégration sociale défaillante et de leurs privilèges financiers. Ainsi, les nouveaux arrivés n'avaient «aucun goût pour Berlin-Est», ne voulaient envoyer leurs enfants que dans les coûteuses garderies des élites de l'ouest de la ville et empochaient des indemnités forfaitaires de déménagement et des allocations pour leurs déplacements entre Bonn et Berlin. Des tensions sociopolitiques entre l'ancienne et la nouvelle capitale se manifestèrent dans le scepticisme avec lequel on observait l'établissement jusqu'alors inédit d'une High-Society ${ }^{20}$ à Berlin. On craignait que l'arrivée des Bonnois, par la présence de nouvelles classes moyennes bourgeoises, puisse déformer $l^{\prime}$ « habitus de la ville ${ }^{21} »$ et refouler les milieux de gauche alternatifs. Au cours de ce débat,

16. Rotes Rathaus: appellation de la mairie de Berlin, construite en pierre rouge (note du traducteur).

17. Frankfurter Allgemeine Zeitung, 24 février 2001.

18. Tous les comptes rendus de la presse évoquent l'année 1958 comme dernière date du défilé berlinois. Il est intéressant de noter que des vétérans berlinois responsables du carnaval prétendent qu'un interdit de démonstration ordonné par les Alliés avait conduit à la suspension du carnaval, un argument que les archives et la presse des années 1950 ne corroborent pas.

19. Arnulf Baring, dans Frankfurter Allgemeine Zeitung, 3 novembre 1999.

20. En anglais dans le texte (note du traducteur).

21. Rolf Lindner, «Der Habitus der Stadt - ein kulturgeographischer Versuch », dans PGM. Zeitschrift für Geo- und Umweltwissenschaften, 2/2003, p. 46-60. 
une étiquette culturelle rhénane émergea et «le Rhénan» devint une figure culturelle dont la mentalité s'opposait à celle si différente «du Berlinois». Cette stéréotypisation régionale présente un répertoire culturel qui se prête à la mise en scène d'une sorte de cultural clash $^{22}$ entre Berlinois et Bonnois, qui se rencontrent désormais dans les sphères politiques et sociales de la nouvelle capitale. L'étendue du débat jusque dans l'immédiat quotidien politique est illustrée par l'interdiction de célébrer le carnaval au Reichstag, ordonnée par le président du Bundestag Thierse, avec la justification que le carnaval est une tradition catholique plutôt étrangère au protestantisme austère de la Prusse ${ }^{23}$.

Pendant la discussion sur la légitimité du défilé de rue comme rituel culturel d'établissement des «Bonnois à Berlin », les tensions politiques entre l'ancienne et la nouvelle capitale se déplacèrent dans la sphère de la politique culturelle. Tandis que d'anciens Bonnois issus de milieux parlementaires qui comptaient célébrer le carnaval expliquaient vouloir perpétuer « la culture et la tradition politico-carnavalesque des 50 années bonnoises de gouvernement allemand à Berlin ${ }^{24} »$, cette invasion était perçue, du point de vue berlinois, avec méfiance et appréhension: "À présent que "les Bonnois” prennent leurs aises à Berlin, le moment de l'offensive carnavalesque est venu ${ }^{25}$.»Pour les voix critiques, le carnaval d'origine rhénane était une importation forcée et artificielle de Bonn «aussi étrangère à la Prusse que la cathédrale de Cologne au capitaine de Köpenick ${ }^{26}$ ». Des voix plus modérées établissaient le pronostic suivant: « Un peu de carnaval et un peu de République de Bonn font du bien à la capitale fédérale», mais la coutume doit «bien entendu rester en Rhénanie, tout comme le Laupenpieperfest et la Love Parade doivent demeurer à Berlin ${ }^{27} »-$ vouloir implanter le carnaval ici ne peut être qu'inauthentique. La question de savoir si «les Bonnois » ont le droit de venir avec leurs coutumes ou non prit la forme d'un choc des cultures entre la Prusse et la Rhénanie. Les adversaires argumentaient que le genre ne jouissait en Prusse d'aucune «tradition ancienne et historique ${ }^{28} »$. La tentative de transposer le carnaval à Berlin ne pourrait qu'échouer, car il lui avait toujours manqué la force subversive dont il avait disposé en Rhénanie contre l'impopulaire force d'occupation prussienne d'autrefois ${ }^{29}$. Tandis que les adversaires du carnaval introduisaient sur le champ de bataille histoire, tradition et mentalité, ses partisans avaient recours au discours du multiculturalisme. Parce que Berlin est une ville tolérante et ouverte sur le monde et aux cultures étrangères et que sa population a non seulement développé un goût pour les «doners et les falafels ${ }^{30}$ », mais aussi depuis longtemps pour les défilés exotiques tels que le Carnaval des cultures, les conditions préalables étaient réunies pour que le défilé du

22. En anglais dans le texte (note du traducteur).

23. Wolfgang Thierse dans le Tagesspiegel, 2 mars 2000.

24. Tagesspiegel, 27 octobre 1999.

25. Ibidem.

26. Frankfurter Rundschau, 21 février 1998.

27. L’ancien premier ministre de Rhénanie-du-Nord-Westphalie, Jürgen Rüttgers, dans la Tageszeitung, 4 mars 2000.

28. Sebastian Haffner, dans Frankfurter Allgemeine Zeitung, 24 février 2001.

29. Frankfurter Allgemeine Zeitung, 26 février 2001.

30. Tageszeitung, 16 février 2001. 
carnaval devienne une solide institution: "Et s'il sont prêts à adopter les cultures africaines, pourquoi devraient-ils alors dédaigner la culture rhénane ? $^{31}{ }^{\prime}$

Les propriétaires du bistro «Mission permanente » s'approprièrent ce discours pour leur grande campagne de promotion du nouveau défilé du carnaval berlinois dans la presse régionale et nationale. Aux positions contre le défilé dont l'argumentation faisait appel à la tradition et à l'identité régionales évangéliques et prussiennes, ils opposaient l'image de la ville cosmopolite et multiculturelle et plaçaient le défilé dans le contexte d'autres grands événements tels que la Love Parade et le Carnaval des cultures. Il s'agissait d'une stratégie de légitimation qui présentait le carnaval classique non plus comme un rituel envahissant de l'établissement « des Bonnois », mais comme un rituel d'intégration « des Rhénans » dans une ville ouverte sur le monde. Ces acteurs élevaient la culture rhénane au statut de «culture privilégiée » d'une minorité comparable à d'autres groupes immigrants comme les Turcs, à la culture desquels la ville tolérante de Berlin s'était finalement aussi habituée.

La tentative de rétablir le carnaval public, conçue par les nouvelles élites économiques comme une campagne publicitaire efficace appuyée sur l'interaction entre politique et médias, va de pair avec la recherche d'une forme de carnaval appropriée pour Berlin. Ceci se produit par le recours à des cultural performances actuelles de la part de la société d'immigration et aux stratégies de mise en scène courantes des grandes villes, qui soulignent leur diversité multiculturelle et leur ouverture cosmopolite. Le concept des nouveaux promoteurs du carnaval s'approprie les objectifs de la festivalisation, à savoir attirer dans la ville des touristes avec de grands événements bien médiatisés et créer un climat de divertissement détendu pour stimuler la consommation. Le carnaval classique doit en même temps attirer un public de classe moyenne dont le style de vie diffère sensiblement de celui des publics de festivals berlinois tels que le Carnaval des cultures et la Love Parade pour ainsi combler une prétendue lacune dans le marché des événements culturels de la grande ville. Selon ses promoteurs issus de l'industrie de la gastronomie-spectacle rhénane, le nouveau carnaval doit être compatible avec la « société des loisirs » en « sortant de sa réserve et en prenant la rue», et ainsi offrir à de larges couches sociales l'occasion de s'adonner à un jeu de rôle costumé. Les protagonistes de la nouvelle campagne de promotion du carnaval se démarquent ainsi résolument de la conception traditionnelle des associations de carnaval locales que l'on retrouvait dans l'est comme dans l'ouest de Berlin avant la chute du mur et le déménagement du gouvernement. Bien que les protagonistes originaires de Bonn ne veuillent pas se mêler de la préservation des coutumes propres aux associations locales, ils en sont tout de même dépendants, tant en ce qui concerne l'organisation que la différenciation du carnaval classique des défilés de rue déjà établis à Berlin. En revanche, le carnaval offre à la vingtaine d'associations berlinoises la possibilité de faire leur promotion et leur propre mise en scène, possibilité inexistante dans les décennies précédentes, car la publicité médiatique, les ressources financières et l'appareil organisationnel leur faisaient défaut. Une partie de ces associations avaient déjà été fondées à l'époque de l'après-guerre. Elles insistent désormais sur le fait qu'il existait bien avant

31. Frankfurter Allgemeine Zeitung, 26 février 2001. 
l'arrivée « des Bonnois » un carnaval berlinois original. La nouvelle tentative leur donne l'occasion de renouer avec l'histoire locale du carnaval et les met au défi de redéfinir et réformer la «tradition » en ayant recours à des coutumes ancrées historiquement. Ce sont précisément ces « vieilles» élites urbaines qui ont échoué dans la mise en scène du défilé au cours des années 1950. Nous aborderons dans la rétrospective historique qui suit les causes de cet échec.

\section{Mises en scène manquées : le carnaval berlinois de l'après-guerre}

Tout commença en 1949 par une extraordinaire assemblée constitutive réunie dans la tour émettrice de radio de Berlin. Quatre commerçants, un restaurateur, le maire de l'arrondissement Berlin-Kreuzberg, un médecin et un humoriste de Cologne s'étaient retrouvés pour stimuler le système circulatoire économique d'un «Berlin souffrant d'insularité » par un assaut de diverstissement carnavalesque. Leur appel «à tous les commerçants du Grand Berlin ${ }^{32} »$ proclamait ce qui suit:

À Cologne sur le Rhin, Munich, Paris, Londres, New York, Rome, Nice, dans toutes ces villes on célèbre le carnaval en grand. Mais Berlin est toujours resté dans l'ombre avec son carnaval. Les commerçants berlinois ont malheureusement trop peu saisi l'excellente occasion qui se présente actuellement à l'ensemble du monde des affaires. Le carnaval n'est pas seulement une fête populaire, mais un événement commercial de premier rang (...) L'homme d'affaires berlinois ne peut laisser cette chance lui échapper encore plus longtemps (...) Berlin pourra dire avec raison le 11 novembre 1949 : « une vie nouvelle renaît des ruines! ${ }^{33}$

On fonda à cette fin la «Grande société berlinoise du carnaval Rheingold», un club de dignitaires qui comptait au début quelque 400 membres issus des secteurs de la gastronomie, de l'industrie, du commerce et de la vie culturelle. L'intérêt des gens d'affaires était de libérer la ville de l'isolation et de la situation économique critique auxquelles elle avait succombé en conséquence de la guerre, de la division et du blocus. Cette classe mercantile qui venait de profiter de la réforme monétaire avait conçu le carnaval pour Berlin comme une campagne publicitaire devant stimuler la classe moyenne et la consommation. D'un point de vue sociopolitique, le projet de carnaval visait la création d'emplois ainsi que l'amélioration de l'humeur d'une population berlinoise frappée par la faim, la pauvreté et le chômage. Le patron de la campagne de promotion du carnaval, le maire Kressmann du district de Kreuzberg, fit remarquer:

Cinq ans après la fin de la guerre, un nouvel optimisme verra le jour dans cette ville tant éprouvée et on donnera à ses habitants amplement l'occasion de se livrer à une saine ironie de soi. Une grande fête populaire est prévue dans les rues de Berlin. Les plus jeunes comme les plus vieux, les riches comme les pauvres berlinois oublieront leurs soucis à l'occasion de ce carnaval et porteront en tombe cinq années tracassantes d'après-guerre ${ }^{34}$.

32. Stadt-Telegraph, 30 août 1949.

33. Reproduction dans Hans Schubert : « Karneval in Berlin - Zwischen Teilung und Wiedervereinigung der Stadt », (1948/49-1989/90), Berlin 2001 (manuscrit non publié), p. 7.

34. Die Welt, 30 août 1949. 
En tant que grande fête, le carnaval semblait être le véhicule approprié pour détendre les tensions sociales et devait en même temps être mis à contribution pour la réalisation déterminée d'intérêts économiques et politiques, objectifs succinctement exprimés par le slogan « réunir efficacement plaisir et création d'emploi ${ }^{35}$ ».

D'un point de vue culturel, la campagne de promotion du carnaval peut être interprétée comme une tentative de revaloriser symboliquement Berlin pour donner à l'ancienne capitale une nouvelle conscience de soi. Il s'agissait de surmonter mentalement la guerre, la défaite, la partition de l'Allemagne et la perte du statut de capitale, ainsi que de refouler collectivement le sentiment de culpabilité et de triompher du stigmate de l'ancienne capitale du Reich, source quelques années plus tôt de violence, d'extermination et de destruction. Le recours à la fête populaire et au carnaval, deux formes de la culture festive populaire qui se prêtent à la mise en scène et à la médiatisation, n'était pas une simple stratégie politico-culturelle de marketing urbain orienté vers l'extérieur de la ville. Il s'agissait en même temps d'une tentative interne de reconstruire l'estime de soi de la population en essayant de produire une forme de rituel communautaire pour qu'elle puisse surmonter les fardeaux du passé et du présent par le truchement de la célébration collective et de l'autoréflexion ironique. Le carnaval et la fête populaire, deux formes d'expression populaires et genres de l'industrie moderne du divertissement, sont fonctionnalisés dans le but de créer une communauté imaginaire dans la ville divisée en renouant par leur intermédiaire avec la culture berlinoise du loisir de l'avant-guerre, les bals et les fêtes de la bière. De plus, le concept nationaliste de la communauté résonne dans le champ sémantique de la fête populaire, et le carnaval suggère l'idée d'une égalité temporaire transcendant l'âge et les classes sociales. On assimile dans ce rituel, exactement selon le concept de drame social de Victor Tunrner ${ }^{36}$, les cicatrices, pour le dire pathétiquement, de la capitale tombée du Reich ainsi que les blessures de la crise sociale actuelle. L'image ternie de la ville doit du coup être repolie, pour la sortir de la crise et la conduire à l'essor économique. Il est donc permis d'interpréter hors de tout doute la tentative d'introduire le carnaval à Berlin pendant l'après-guerre comme une stratégie de politique économique et sociale exécutée par une nouvelle élite urbaine dans le but d'initier une sorte de rituel de transition pour une ville entière et sa population, pour les transposer collectivement dans cette phase de prospérité, qu'on appellera rétrospectivement l'ère du « miracle économique allemand ».

Le carnaval était appelé à s'émanciper des formes et des traditions de l'Allemagne de l'Ouest et du sud; il ne pouvait ni paraître contraint ni imiter le carnaval rhénan - bref, il devait être berlinois. Et c'est justement là que reposait le problème: mettre sur pied un carnaval signifiait vouloir implanter une forme de culture festive populaire, traditionnelle ailleurs, mais pas à Berlin, où un tel spectacle, même avant la guerre, n'avait jamais existé. Deux tentatives, en 1871 et en 1911, avaient échoué à la suite de critiques massives de la part des spectateurs; en 1871, on avait même mitraillé le prince du carnaval de crottin de

35. Ibidem.

36. Victor Turner, «Soziale Dramen und Geschichten über sie», dans l'ouvrage du même auteur: Vom Ritual zum Theater, Francfort-sur-le-Main/New York, 1989, p. 95-140. 
cheval. Le recours au carnaval était donc attaché à un problème d'authenticité qui rendait nécessaires des stratégies de mise en scène particulières. Afin de résorber le problème, c'est-à-dire de conférer au carnaval plausibilité et crédibilité et de mobiliser les masses, il fallait adapter la forme adoptée du carnaval aux spécificités locales. Comment essaya-t-on d'inventer, dans les années 1950, un carnaval typiquement berlinois? Au départ, la presse rendait encore compte du club de dignitaires avec amusement:

Un historien estimait [que] l'esprit de Glassbrenner, Fontane, Simmel, Ringsnatz, que bref tout ce qui avait été en jachère à la sombre époque allait ressusciter. Les fêtes costumées dans de vieux bistros et des estaminets de bière blanche, les soirées de carnaval entre hommes ou femmes membres des associations les plus variées, proposait-il. Un grand défilé de fête dans les rues hivernales de Berlin, peuple et chars en train avec des personnages berlinois tels que Nante, Gustave d'acier, Walter le consolateur, les jeunes policières et les femmes des décombres $^{37}$. On devrait organiser des fêtes pour enfants dans tous les quartiers, suggérait un autre (...). Au lieu de «Réjouissez-vous de la vie», il faudrait composer une nouvelle chanson de carnaval. Auteurs de rengaines, approchez! Un des participants, un immigré rhénan, était tout feu tout flamme. En plus des bals masqués, il proposait une délégation qui irait féliciter le maire et, le mercredi des cendres, une séance de lavage des portefeuilles sur les rives de la Panke. Il décrivait le carnaval de sa ville natale où l'on distribuait aux bandes d'enfants bretzels et oranges depuis les chars allégoriques. Un journaliste répliqua, l'inévitable cigarette au coin de la bouche, qu'à Berlin, naturellement, il faudrait distribuer des saucisses et des cigares ${ }^{38}$.

Professeurs de danse, compositeurs, chansonniers, librettistes et éditeurs furent invités dans le cadre d'un concours à créer de "véritables» danses et chansons berlinoises pour ainsi « ouvrir la voie à de nouvelles formes folkloriques et artistiques». On espérait de la sorte « enfin contribuer mélodieusement et rythmiquement à une gaieté berlinoise originale ${ }^{39} »$.

Une « identité berlinoise » se constitue à partir d'un fonds de caractéristiques emblématiques. L'Invention of Tradition ${ }^{40} s^{\prime}$ accomplit par le recours au « symbolisme indigène ${ }^{41}$ », c'est-à-dire que des signes, explicitement liés à Berlin, sont choisis pour être fusionnés dans une unité caractéristique de la représentation culturelle. La «culture rhénane» sert d'émulsifiant à ce bricolage folklorique. Dans l'esprit des initiateurs du carnaval, il semblait possible de réunir en les additionnant les uns aux autres divers éléments du fonds emblématique local pour créer un carnaval berlinois qui ne serait pas fondé sur une «coutume traditionnelle». On cita à l'appui tous ces représentants du patrimoine berlinois qui à diverses époques avaient contribué à l'édification de la spécificité culturelle de Berlin:

37. Trummerfrauen: femmes qui dans l'après-guerre déblayaient les décombres laissés par les bombardements alliés (note du traducteur).

38. Stadt-Telegraph, 28 juin 1949.

39. Die Welt, 30 août 1949.

40. En anglais dans le texte (note du traducteur).

41. Richard L. Wohl et Anselm L. Strauß, «Symbolic Representation and the Urban Milieu», dans AJS, vol. 63,1958, p. 523-532. 
Glassbrenner, Hofmannsthal et Zille ${ }^{42}$. Ils avaient créé ces emblèmes littéraires et picturaux qui appartiennent au répertoire de la mentalité spécifiquement berlinoise. En tant que figures populaires issues des milieux prolétaires et petits-bourgeois, des personnages berlinois originaux tels qu'Eckensteher Nante ${ }^{43}$ ou Harfenjule ${ }^{44}$ incarnent à la perfection l'idéal type de l'habitus de la ville. Ils représentent de façon légendaire les cultures des "petites gens », l'âpre impertinence et le sens de la répartie - des stéréotypes psychologiques tels que la "grande gueule berlinoise » et l' « humour berlinois » - qui ont été reproduits par d'innombrables créations de l'industrie culturelle des $\mathrm{XIX}^{\mathrm{e}}$ et $\mathrm{XX}^{\mathrm{e}}$ siècles comme emblèmes culturels de Berlin. Le recours à de pareils segments transcendants d'une «caractériologie populaire ${ }^{45}$ de la ville évoque des époques et des périodes en apparence peu chargées, telles que le Xix ${ }^{\mathrm{e}}$ siècle, la révolution industrielle allemande et les années 1920. Un romantisme bourgeois et une transfiguration de la culture populaire urbaine évitant le nazisme et la guerre sont inhérents au mélange de la culture festive prolétarienne/ petite-bourgeoise, de la culture associative bourgeoise et de la culture urbaine du loisir. Mais au bout du compte, le concept de mise en scène du « soi », selon lequel la population berlinoise se reconnaîtrait dans le miroir d'une représentation carnavalesque et traditionnelle faisant fi de ses sensibilités réelles, échoua.

D'entrée de jeu, la majorité des quotidiens critiquent virulemment la campagne de promotion du carnaval. Les attaques s'adressent à la classe politique et aux commerçants, auxquels on reproche d'instrumentaliser le carnaval à leurs propres fins. «Il faut le dire, les choses ne sauraient être plus insensées. On tient la dragée haute aux Berlinois et on voudrait ensuite en faire des fous. Tels sont les soucis d'un maire berlinois ${ }^{46}$.» Le comité d'alimentation de la Kommandantur de Berlin-Ouest, dont faisait aussi partie le maire Kressmann de Kreuzberg, venait tout juste de stopper la distribution de cartes de rationnement. Interprété comme une ouvre de bienfaisance douteuse et une mesure de création d'emplois inefficace venue «d'en haut», on soupçonne la campagne de promotion du carnaval de vouloir distraire la population de sa détresse par un divertissement artificiel: des jeux au lieu du pain. C'est surtout la nouvelle élite économique qui est dans le collimateur; elle,

42. Heinrich Zille (1858-1929), graphiste, dessinateur et photographe. Son œuvre, qui est devenue extrêmement populaire, représente la vie et le milieu du prolétariat berlinois au tournant du $\mathrm{xx}^{\mathrm{e}}$ siècle dans une optique de critique sociale à la fois tendre et ironique. Le recueil de photographies « Mein Milljöh » et les cycles «Hurengespräche» et «Berliner Luft» sont particulièrement fameux.

43. Personnage d'Anton Glassbrenner devenu un classique de l' « humour berlinois » avec le livre Berlin wie es ißt und trinkt, Berlin, 1932. L'essayiste, critique de théâtre et satiriste était considéré comme un porteparole littéraire «du petit peuple ouvrier», comme le «Zille littéraire». Son «Typenschau des Berliner Volkes», une nouvelle dialogique, établit un nouveau genre littéraire. Sa facétie la plus connue est «Der ächte Eckensteher Nante» (dans Berliner Volksleben, Leipzig, 1847), une satire, qui, imitant les modèles viennois, dénonce la violence policière de l'État prussien. Pendant le Vormärz (période historique entre 1815 et la révolution de 1848 , n.d.t), ses écrits, bannis plus tard, connurent des tirages élevés avec près de 200 réimpressions et contrefaçons.

44. Luise Nordmann (1829-1911), chanteuse qui gagnait sa vie en chantant et en jouant de la harpe dans les cours arrière de Berlin. Figure du " personnage original berlinois digne de pitié ».

45. Lindner, $1999 \mathrm{~b}$.

46. Berlin-Express, 29 août 1949. 
qui, dans la foulée de la réforme monétaire, avait trouvé la prospérité pratiquement du jour au lendemain, tandis que la population au large souffrait toujours de la faim et du chômage. Au divertissement frivole des nouveaux riches, on oppose la détresse sociale des masses : avec le défilé du carnaval, «les profiteurs de la réforme monétaire veulent se défouler de la colère qu'ils ressentent vraisemblablement en raison des 260000 chômeurs, des dizaines de milliers qui doivent quitter leur logement avant Noël et de la conjecture économique des secteurs ouest de la ville dont ils ne sont pas satisfaits [...]. Que les bénéficiaires de la réforme monétaire célèbrent leurs fêtes comme ils le veulent. Leur carnaval aussi s'avérera être une bêtise ${ }^{47}$.»

On attaque aussi l'intention du comité organisateur de faire défiler 32 figures historiques « témoins de l'humour berlinois ${ }^{48}$ ». Parfois la critique exprimée dans les médias se transforme en véritable combat pour ces emblèmes de la ville. L'utilisation de ces figures populaires, incarnations du mode de vie de ceux «d'en bas » pour servir les fins politiques de ceux d' "en haut », constituait sans doute une provocation particulière. On jugea cyniquement anachronique l'utilisation de caricatures de Glassbrenner ou de Zille, qui tirèrent leur potentiel satirique des milieux prolétariens et petits-bourgeois et qui avaient toujours critiqué les autorités et la misère sociale, par des politiciens qu'on tenait responsables de la crise sociale et économique de la ville ainsi que du fossé croissant entre riches et pauvres depuis la réforme monétaire:

On n'a de la peine que pour les personnages originaux de Berlin dont on abuse. Pour le père Zille aussi. Il se retournerait dans sa tombe s'il savait en quelle compagnie son nom est prononcé. Car il existe aussi des fous dangereux (...). Leur place n'est pas dans une société de carnaval, mais dans une camisole de force ${ }^{49}$.

Huit fois, de 1952 à 1959, le carnaval a défilé dans le secteur ouest de la ville de Berlin divisée. Les commentaires de presse oscillaient entre la critique ouverte et la critique retenue. Les premières années, on constata que le défilé attirait certes beaucoup de spectateurs, mais il n'en reste pas moins qu'il « [demeurait] un squelette, qui aurait tout aussi bien pu passer dans les rues à toute autre occasion, un spectacle auquel on assistait sans véritable participation ${ }^{50} »$. En prélude à chaque nouvelle édition du carnaval, les organisateurs soulignaient que le carnaval serait «vrai» cette fois-ci.

Déjà en 1954, 200 artistes et acteurs sans emploi défilèrent déguisés en personnages berlinois. L'écho médiatique n'en demeura pas moins négatif, même après qu'on eut remplacé les chars publicitaires par des chars allégoriques satiriques persiflant la politique du jour : division de l'Allemagne, le pont aérien durant le blocus de Berlin, le chancelier Adenauer, la «surtaxe d'urgence pour Berlin» ou le magistrat de la ville. Pourtant, en 1957, malgré 200000 spectateurs, l'atmosphère ne s'était guère égayée. Les médias mirent en évidence l'aspect artificiel du défilé et s'attaquèrent à son caractère militaro-prussien. On

47. Berliner Zeitung, 13 novembre 1949.

48. Stadt-Telegraph, 30 août 1949.

49. Tribüne, 31 août 1949.

50. Morgenpost, 20 février 1953. 
dit que le signal de départ du défilé donné par le président berlinois du carnaval, «ô fous, réjouissez-vous de la vie», avait plutôt sonné comme un "garde-à-vous » ${ }^{51}$.

En 1959, le carnaval est l'objet de la plus dure critique de la part des médias. Des grands titres comme «Défilé du Lundi des roses décevant - il n'y avait guère de quoi rire $^{52} »$ notent certes que 350000 spectateurs s'étaient déplacés, mais que les Berlinois ne s'étaient guère enthousiasmés. On note aussi que les figures en carton de nature généralement politique avaient eu un effet plus amer que comique. «Lorsque finalement une plantureuse dame en carton présenta chaque moitié de son décolleté avec les inscriptions "Bonn" et "Berlin" sous la devise "Les deux vont ensemble" », les Berlinois perdirent pour de bon toute envie de rire ${ }^{53}$. Afin d'étayer le caractère artificiel du défilé, on s'attarda particulièrement sur le comportement des responsables du carnaval; ici aussi se fait entendre la critique de la mise en scène de soi par une élite urbaine dont la campagne promotionnelle exhalait un flair de propagande: au cours du défilé d'une durée de quatre heures, «ces messieurs n'ont pas cessé d'alimenter la joie de l'intérieur, comme ont pu en témoigner un grand nombre de bouteilles de $\operatorname{cognac}{ }^{54}$ ».

Pendant les années 1950, l'éternelle objection de la presse au carnaval argumentait que la «mentalité rhénane» ne pouvait être transplantée à Berlin. On esquissait ainsi des exemples illustrant l'incompatibilité de la mentalité «berlinoise», exemples issus d'une caractérisation ethnique essentialisée: «L'humour pétillant du Rhénan ne s'accorde pas avec le caractère froid et réservé du Berlinois. Son humour est d'une autre nature ${ }^{55}$.» On opposait aussi au carnaval imposé par le haut une culture festive locale au fondement moins stéréotypé et mieux ancré dans l'histoire culturelle et sociale de Berlin. Cette «tradition berlinoise» était dérivée de formes d'expression propres au divertissement populaire, des fêtes costumées des " petites gens » à l'époque de Guillaume II, en passant par les «nuits tumultueuses de bal au tournant du siècle» jusqu'à la " grande époque de la danse débridée » de la République de Weimar ${ }^{56}$. Le topos de la résistance résonnait dans cet inventaire de la culture du loisir urbaine selon lequel «le Berlinois [accepte certes tous les ordres] de l'autorité, mais jamais d'explosions de joie limitées dans le temps ${ }^{57}$ ». D'autre part, on affirmait l'existence d'une tradition locale qui présentait la caractériologie d'un habitus prolétaire/petit-bourgeois comme typique de Berlin.

En résumé, il apparaît que des médias construisirent à l'aide de contre-exemples de la «mentalité berlinoise » un discours de l'authenticité pour combattre avec force la stratégie d'acculturation mise en œuvre par l'élite urbaine. La mixture additive de stéréotypes des mentalités d'ordre populaire-folklorique et de persiflage politique orchestrée par les organisateurs du carnaval était régulièrement déconstruite par les médias comme une stratégie de mise en scène populiste. La mauvaise presse que reçut le carnaval en 1959 eut

51. Die Welt, 3 mars 1957.

52. BZ, 9 février 1959.

53. Süddeutsche Zeitung, 9 février 1959.

54. Ibidem.

55. Berliner Zeitung, 13 novembre 1949.

56. Feuilles d'images berlinoises dans Der Kurier, 8 février 1959.

57. Ibidem. 
pour conséquence que de grandes entreprises, parmi lesquelles une importante brasserie, mirent un terme à leur commandite, privant ainsi le carnaval d'une source essentielle de financement. L'assistance nombreuse ne suffisait pas à ancrer le carnaval dans la scène locale. Le carnaval se retira dans les arrière-chambres de bistros et dans des salles d'hôtel ${ }^{58}$. À plusieurs égards, le carnaval de rue s'était révélé un anachronisme dans les années 1950.

\section{BILAN}

Distances dans le temps, il y a eu à Berlin quatre tentatives différentes d'introduire le carnaval de rue dans l'espace public. Trois d'entre elles échouèrent; ce qu'il adviendra de la quatrième reste à découvrir. La dernière tentative se situe dans le contexte du changement de capitale et du transfert de la classe politique rhénane à Berlin.

Une perspective historique comparative sur l'introduction (manquée) du carnaval dans les années 1950 révèle que l'on contesta à l'époque l'importation du carnaval rhénan à l'aide des mêmes représentations des mentalités qu'aujourd'hui. Autre ressemblance, les élites économiques et politiques, en majorité venues de l'extérieur, essayèrent de faire la promotion du carnaval à l'aide d'une campagne d'image culturelle, pensée à la fois comme une intervention stimulante pour l'économie et un véhicule pour apaiser les tensions sociales. L'instrumentalisation par ces élites du patrimoine folklorique urbain et une certaine «typification berlinoise authentique» furent critiquées par les médias comme l'inauthentique mise en scène de soi par une élite économique qui s'était désavouée devant la crise sociale et économique. Il devient particulièrement clair que les médias, en tant qu'instances évaluatrices, ont un rôle considérable à jouer dans le succès ou l'échec de l'implantation de Public Performances dans l'espace public ${ }^{59}$.

L'Invention of Tradition s'accomplit aujourd'hui dans le contexte de la culture contemporaine de l'événement et de l'interaction entre les anciennes et nouvelles élites urbaines. Dans la tentative renouvelée d'établir le carnaval, deux stratégies de mise en scène prêtent au carnaval d'origine rhénane un caractère berlinois : les promoteurs originaires de Rhénanie tentent d'établir le carnaval en ayant recours à l'image multiculturelle de Berlin et le placent dans le contexte de la culture des grands événements caractéristique des grandes villes tels que la Love Parade et le Carnaval des cultures. Il s'agit d'une stratégie de légitimation qui ne laisse plus paraître le carnaval classique comme un rituel d'établissement « des Bonnois » venus occuper l'espace social, mais qui plutôt le présente comme un rituel d'intégration «des Rhénans » dans une ville ouverte sur le monde. En revanche, les acteurs locaux, c'est-à-dire les associations de carnaval longtemps établies à Berlin, ont

58. Un carnaval pour dignitaires d'associations s'établit dans le secteur ouest de Berlin, qui recrutait ses membres parmi les réseaux de propriétaires de brasseries, d'administrateurs, de gens d'affaires et de politiciens locaux. Dans le secteur est de la ville, des clubs de carnaval se formèrent au sein des entreprises étatiques de la RDA.

59. La réflexivité est un élément constitutif de toute cultural performance ou, plutôt, de tout rituel ; l'événement, dans la répercussion et l'évaluation de l'ante-mortem et du post-mortem des cultural performances, prend essentiellement forme tant en fonction de sa perception que de sa reprise (Ronald L. Grimes, «Ritual Criticism : Case Studies in its Practice. Essays on the Theory», Columbia, South Carolina, 1990). 
recours à une coutume enracinée historiquement: la campagne du carnaval leur donne l'occasion de renouer avec l'histoire locale du carnaval berlinois. Il s'avère que «festival» et «coutume» ne s'excluent pas mutuellement, qu'ils sont plutôt deux modes de représentation et de mise en scène excellents pour la publicité qui sont combinés avec succès, sont compatibles avec les modes de mise en scène courants de l'image politique urbaine et soulignent le caractère métropolitain de la capitale « représentative » à l'aide de références à la diversité multiculturelle. Dans la tentative contemporaine de rétablir le carnaval, les médias servent encore de voix puissante qui accompagne et évalue l'événement. Mobilisés sélectivement par les gastronomes originaires de Bonn et leurs réseaux, les médias ont contribué à donner une saveur exotique au carnaval, une nouveauté dans le cadre du déménagement du gouvernement, qui, dans le contexte du débat sur le carnaval, est à son tour devenu un événement culturel.

En conclusion, et au-delà du cas concret, on s'aperçoit que le fonds imaginaire de la culture locale, c'est-à-dire le fonds d'images historiquement saturées, de représentations et de significations du lieu concret, n'est pas en soi un dispositif décisif vers lequel doivent s'orienter les campagnes politco-culturelles de promotion d'événements des élites urbaines pour réussir. D'autre part, de telles stratégies de marketing de mise en scène publicitaire de la ville ne peuvent venir exclusivement d' "en haut». La capacité des Public Performances à s'établir ou non dans le cadre des processus de transformation de la ville postfordiste est soumise à un processus plus complexe de négociations entre les groupes d'acteurs participant à la préparation et à l'évaluation d'événements performatifs. Ce résultat vaut généralement pour les rituels au sein de sociétés pluralistes et complexes.

Ainsi, en ce qui concerne le carnaval de Berlin, sa capacité à intégrer durablement le fonds de la représentation culturelle du «nouveau Berlin » en tant que grand spectacle reste à déterminer. Il est possible que le carnaval devienne plus créatif, pluriel et ouvert aux formes de représentation alternatives ou encore plus satiriques, en ce qui a trait à la « République de Berlin ». Peut-être aussi aura-t-il encore une fois disparu de la scène dans quelques années.

\section{RÉSUMÉ}

Se penchant sur les multiples tentatives de réintroduction du carnaval de rue à Berlin, cet article s'intéresse à la volonté de reconstruire Berlin comme lieu et théâtre de l'Allemagne réunifiée en opérant certaines transformations symboliques issues de divers débats sur la façon dont la ville peut devenir cette capitale. À travers une perspective comparative historique, l'article analyse les campagnes de marketing politico-culturelles de réinvention ainsi que les diverses mises en scène innovatrices de performances culturelles pour donner au carnaval d'origine rhénane un caractère berlinois, dans deux contextes historiques et sociopolitiques différents, soit celui des années 1950 et celui des années 2000. L'article démontre que la capacité de ces mises en scène à s'établir dans le cadre des processus de transformation de la ville postfordiste est soumise à un processus complexe de négociations entre les groupes d'acteurs participant à la préparation et à l'évaluation de telles performances symboliques. 


\section{ABSTRACT}

Focussing on the numerous attempts to reintroduce the street carnival in Berlin, this paper studies the desire to reconstruct Berlin as the site and theatre of a reunified Germany by conducting certain symbolic changes stemming from various debates concerning the way in which the city can truly become that country's capital. Through a comparative historical perspective, the paper analyses Berlin's political and cultural marketing campaigns to reinvent itself as well as the various innovative scenarios for cultural performances to give a Berlin character to the original Rhine-based carnival, in two different historical and socio-political contexts, namely that of the 1950 s and that beginning in the year 2000 . The paper shows that the ability of these scenarios to implant themselves within the transformation processes of the post-Fordist city is subject to a complex process of negotiations between the groups of actors participating in the preparation and evaluation of such symbolic performances.

\section{RESUMEN}

Examinando las múltiples tentativas de reintroducción del carnaval de la calle en Berlín, este artículo se interesa a la voluntad de reconstruir Berlín como lugar y teatro de la Alemania reunificada operando algunas transformaciones simbólicas resultantes de distintos debates sobre la forma en que la ciudad puede convertirse en esta capital. A través de una perspectiva comparativa histórica, el artículo analiza las campañas de comercialización político-culturales de reinvención así como las distintas puestas en escena innovadoras de hechos culturales para dar al carnaval de origen renano un carácter berlinés, en dos contextos históricos y sociopolíticos diferentes, el de los años 1950 y el de los años 2000. El artículo demuestra que la capacidad de estas puestas en escena para establecerse en el marco de los procesos de transformación de la ciudad post-fordista está sometida a un proceso complejo de negociaciones entre los grupos de actores que participan en la preparación y en la evaluación de tales hechos simbólicos.

\section{BIBLIOGRAPHIE}

Bailey, F. G. (1996), «Cultural performance, authenticity, and second nature» in D. Parkin et al. (Éds.), The Politics of Culture Performance, Oxford, p. 1-17.

BARING, A. (1999), dans le Frankfurter Allgemeine Zeitung, 3 novembre.

Baumann, G. (1992), «Ritual implicates 'Others' : Rereading Durkheim in a plural society », in D. De Coppet (Éd.), Understanding Rituals, Londres, Routledge.

Becker, F. et B. Binder (2001), «'Bühnen der Macht': Stadtethnologische perspektiven auf die hauptstadtwerdung Berlins », Humboldt Spektrum, vol. 8, n 3-4, p. 112-119.

BeCKer, F. et B. Binder (2003), «Hauptstadt-Rituale. Cultural performances und ihre autorisierung im öffentlichen raum », E. Fischer-Lichte et al. (Éds.), Ritualität und Grenze (Theatralität vol. 5), Tübingen, p. 224-244.

BerLiner VolksLeben, Leipzig, 1847

BerLiner Zeitung, 13 novembre 1949.

BERLIN-EXPRESS, 29 août 1949.

Boyer, M.C. (1995), «The great frame up. Fantastic appearance in contemporary spatial politics» in H. LiggetT et D. C. Perry (Éds.), Spatial Practices, Thousand Oaks (CA.), Sage, p. 81-109.

Bz, 9 février 1959.

Conen, A. (1993), Masquerade Politics. Explorations in the Structure of Urban Cultural Movements, Oxford \& Providence, Berg. 
Der Kurier (1959), Feuilles d'images berlinoises, 8 février.

Die Welt, 30 août 1949.

Die Welt, 3mars 1957.

Frankfurter Allgemeine Zeitung, 24 février 2001.

Frankfurter Allgemeine Zeitung, 26 février 2001.

Frankfurter RUNDSChau, 21 février 1998.

GeERTZ, C. (1993), «Deep play: Bemerkungen zum balinesischen hahnenkampf», in Dichte Beschreibung, Francfort sur le Main, Suhrkamp, p. 202-260.

Grimes, R. L. (1990), Ritual Criticism: Case Studies in its Practice. Essays on the Theory, Columbia, University of South Carolina Press.

HaffNer, S. (2001), dans le Frankfurter Allgemeine Zeitung, 24 février.

Handelman, D. (1990), Models and Mirrors. Toward an Anthropology of Public Events, Cambridge, Cambridge University Press.

Häussermann, H. et W. Siebel (1993), «Die politik der festivalisierung und die festivalisierung der politik. Große ereignisse in der stadtpolitik», in H. Häubermann et W. Siebel (Éds.), Festivalisierung der Stadtpolitik, Leviathan, édition spéciale 13, Westdeutscher, Verlag, p. 7-32.

Hobsbawn, E. (1993), «Introduction: Inventing traditions» in E. Hobsbawn et T. Ranger (Éds.), The Invention of Tradition, Cambridge, p. 1-15.

Hobsbawn, E. (1998), « Scènes du pouvoir. De la mise en scène du 'quartier gouvernemental' comme espace de représentation ", projet de recherche subventionné par la Deutsche Forschungsgemeinschaft (DFG), Berlin, l'Institut für Europäische Ethnologie de l'Université Humboldt à Berlin.

Linder, R. (1998), «Stadtkultur », in H. Häubermann (Éd.), Großstadt. Soziologische stichworte, Opladen, Leske \& Budrich, p. 256-262.

LINDER, R. (1999a), «The imaginary of the city » in Le ministère fédéral de la Science et des Transports et par le Centre international de recherche sur les sciences de la culture (Éds.), The contemporary study of culture, Vienne, p. 289-294.

Linder, R. (1999b), «Die kulturelle textur der stadt », in Das Magazin, Zeitschrift des Wissenschaftszentrums Nordrhein-Westfalen, vol. 10, p. 34-35.

Linder, R. (2003), «Der Habitus der Stadt - ein kulturgeographischer Versuch », PGM, Zeitschrift für Geo- und Umweltwissenschaften, février, p. 46-60.

Ludwig, H. (1932), Berlin wie es ißt und trinkt, Berlin.

Morgenpost, 20 février 1953.

RÜTtGeRs, J. (2000), dans la Tageszeitung, 4 mars.

Schieffelin, E. L. (1998), «Problematizing performance», in F. Hughes-Freeland (Éd.), Ritual, Performance, Media, Londres, Routledge, p. 194-207.

SChUbert, H. (2001), Karneval in Berlin - Zwischen Teilung und Wiedervereinigung der Stadt (1948/49-1989/ 90), contient la reproduction de l'extrait original, manuscrit non publié, Berlin, p. 7.

SINGER, M. (1972), "Search for a great tradition in cultural performances", in When a great tradition modernizes: An Anthropological approach to Indian civilization, Londres et New York, Praeger, p. 67-80.

Stadt-TELegraph, 28 juin 1949.

STADT-TELEgRAPH, 30 août 1949.

SÜDdeUtSCHE-Zeitung, 9 février 1959.

TAgessPiegeL, 27 octobre 1999.

TAGESSPIEgeL, 12 septembre 2000.

TAgESSPIEgEL, 16 février 2001.

TRIBÜNE, 31 août 1949.

TURner, V. (1989), «Soziale dramen und geschichten über sie », in V. Turner (Éd.), Vom ritual zumt theater, Francfort sur le Main et New York, p. 95-140.

Wohl, R. L. et A. L. Strauss (1958), «Symbolic representation and the urban milieu », American Journal of Sociology, vol. 63, March, p. 523-532. 
Wolfgang, T. (2000), dans le Tagesspiegel, 2 mars.

Zukin, S. (1995), The Cultures of Cities, Cambridge (MA.), Blackwell Publishers. 